\title{
Right congenital diaphragmatic hernia - a diagnosis to consider
}

\author{
Ruxandra Darie', Raluca Maria Vlad ${ }^{1,2}$ \\ 1"Grigore Alexandrescu" Emergency Children's Hospital, Bucharest, Romania \\ 2"Carol Davila" University of Medicine and Pharmacy, Bucharest, Romania
}

\begin{abstract}
Objectives. Diaphragmatic hernia is not a common disease. The severity of symptoms may vary depending on the size of defect and the organs involved. A defect on the right side is quite rare. This congenital condition may not be clinically obvious for a long time.

Material and method. We present the case of a patient hospitalised in the Pediatrics Department of "Grigore Alexandrescu" Hospital for unrelated pathology, incidentally diagnosed with right congenital diaphragmatic hernia after the age of three.

Results (case presentation). 3 years 6 months old girl is admitted for persistent cough. An antenatal diagnosis of agenesis of the aortic arch had been set. She was born premature, required intensive care during the neonatal period, one diagnosis being right pulmonary hemorrhage. Postnatal ultrasound examination diagnosed venous duct anomaly. She had a good clinical course with normal growth, normal cardiac and liver functions. The clinical examination was unremarkable. A thoracic X-ray described homogeneous, well-defined opacity in the right lower pulmonary lobe. Abdominal ultrasound confirmed azygos continuation of the inferior vena cava. She underwent antibiotic therapy for pneumonia (7 days) with no change of the thoracic image. Thoracic computed tomography demonstrated Morgagni's hernia with liver thoracic dislocation.

Conclusions. Diaphragmatic hernia is a challenging diagnosis to be made. Although a congenital condition, it may be asymptomatic for a long time and a high degree of suspicion is required.
\end{abstract}

Keywords: diaphragmatic hernia, child, Morgagni

\begin{abstract}
Abbreviations
CDH: Congenital diaphragmatic hernia

CDMH: Congenital diaphragmatic Morgagni hernia
\end{abstract}

\section{INTRODUCTION}

Congenital diaphragmatic hernia $(\mathrm{CDH})$ refers to a defect in the development of diaphragm muscle that leads to protrusion of abdominal viscera into the thoracic cavity, thus interfering with the normal development of the lungs. The condition has two main features: absence/deficiency of the diaphragm due to an incomplete formation of the muscle or incomplete muscularization that leads to eventrations (a thinned part of the diaphragm is elevated) $(1,2)$. The male/female ratio is slightly in favor of men. However, in Morgagni's hernias there is no clear evidence of gender association (3).

$\mathrm{CDH}$ may present as an isolated defect or as part of a syndrome. $2 \%$ of all cases are reported to be familial, transmission being associated with auto- somal recessive, autosomal dominant or $\mathrm{x}$-linked. Fryns syndrome is an autosomal recessive disease which includes $\mathrm{CDH}$, anomalies of the brain, heart, urinary system and hypoplasia of distal digits. Cornelia de Lange syndrome is an autosomal dominant disorder, characterized by the association of particular facial traits, $\mathrm{CDH}$, hirsutism and development delay. Trisomy 13, 18 and 21 are the most common chromosomal abnormalities related to CDH $(2,4)$.

Etiology is unclear and is thought to be multifactorial. There are multiple genetic, environmental and nutritional factors such as defects in vitamin A pathway, low retinol/retinol-binding protein or herbicide (2).

The diaphragm muscle develops during the $8^{\text {th }}$ and $12^{\text {th }}$ week of gestation. Thoracic and abdominal 
cavities are separated by the septum transversum, a membrane populated later with muscle fibers. When organs from the abdomen migrate into the chest cavity, lung growth is poor, resulting in pulmonary hypoplasia, the main complication of $\mathrm{CDH}$ $(4,5)$.

Left side defects are more common (85\% of all cases) and are often associated with herniation of stomach, intestines, liver or spleen into the chest cavity. They are called Bochdalek (posterolateral) hernias. Non-posterolateral hernias are anterior defects of the diaphragm and can appear either on the right or left side, but also in the midline. MorgagniLarrey hernia affects almost $2 \%$ of the newborn population, is located on the right side and consists in herniation of the liver and intestines into the thoracic cavity. Other anterior defects $(2-7 \%)$ include Pentalogy of Cantrell (CDH, abnormalities of the heart), a severe type of hernia and central hernia (a rare condition involving the non-muscular part of the diaphragm) $(1,6)$.

Congenital diaphragmatic Morgagni hernia $(\mathrm{CDMH})$ always has a hernia sac represented by parietal peritoneum or hypoplastic diaphragm. Most of the cases are associated with multiple malformations or chromosomal disorders such as intestinal malrotations, ventricular septal defects, pulmonary artery stenosis or Down syndrome. Most cases of CDMH manifest in adulthood and are rarely diagnosed in the pediatric population. These patients rarely present with intestinal obstruction or strangulation at the beginning. The diagnostic is suspected on plain X-ray and is confirmed by CTscan. Differential diagnosis includes: bronchogenic cysts, congenital cystic adenomatoid malformation, cystic teratomas, pulmonary agenesis (3).

\section{CASE PRESENTATION}

A 3 years 6 months old girl was hospitalized for persistent wet cough. On history, the parents reported multiple upper respiratory tract infections in the last 6 weeks (after starting preschool), with brief periods of improvement after symptomatic treatment. Four days before hospital admission, the patient experienced severe and frequent outbursts of productive cough with shortness of breath. No other clinical signs were associated.

Prenatal outline suspected in the second trimester a life-threatening cardiac abnormality (aortic arch agenesis), which was later ruled out. Due to intrauterine ascites and other obstetrical complications, the patient was born premature at 33 weeks of gestation through $\mathrm{C}$-section with an APGAR score of 6-7. She required intensive care during neonatal period, one diagnosis being right pulmonary hemorrhage (fig. 1). Postnatal ultrasound examination revealed venous duct anomaly (drainage of hepatic veins in the right atrium), mild hypertrophic cardiopathy and ascites.

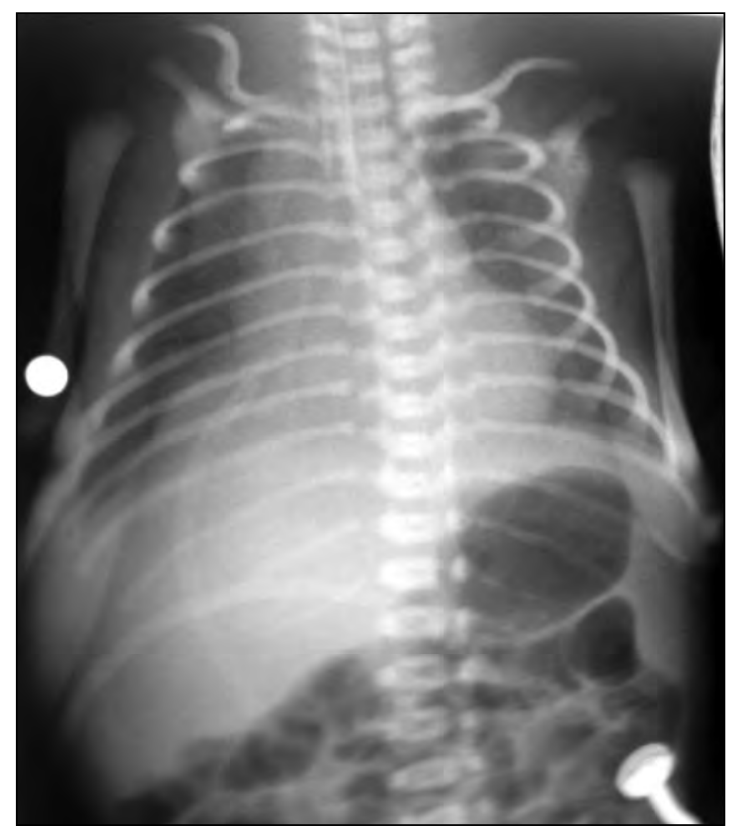

FIGURE 1. Chest $x$-ray from the neonatal period: patient diagnosed with pulmonary hemorrhage

The patient had a good clinical course over the first 3 years of life, with normal growth and normal cardiac and hepatic functions.

At admission, the clinical findings were productive cough, respiratory rate of 24 per minute, with no crackles on lung auscultation, an oxygen saturation up to $99 \%$ and a heart rate of 80 beats per minute. Multiple small cavernous hemangiomas were discovered on the scalp and thorax. Otherwise the clinical examination was unremarkable.

Cell blood count showed normal hemoglobin value, white blood cell count $=19,100 / \mathrm{mm}^{3}$, with a normal leukocyte differential count, and platelets = $320,000 / \mathrm{mm}^{3}$. No inflammatory syndrome was found. Immunological markers, liver and renal functions were normal. Nasal and pharyngeal cultures, gastric lavage for acid-fast bacilli, Mantoux test and bronchial aspirate were all negative.

A chest X-ray was obtained and demonstrated homogenous, well-defined opacity in the right lower pulmonary lobe (fig. 2).

Abdominal ultrasound confirmed complex malformation of the venous duct (abnormal drainage of the left hepatic vein into the right atrium and the right hepatic vein in the vena cava). Echocardiography maintained the diagnosis, revealing azygos continuation of the inferior vena cava. 


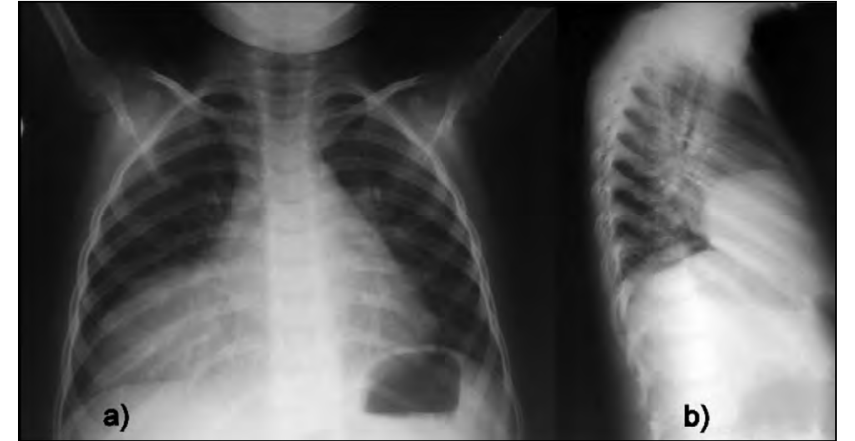

FIGURE 2. Chest x-ray: (a) posteroanterior view, (b) right side view

After clinical, laboratory and imaging considerations, diagnoses established were: medium lobe pneumonia, complex venous duct abnormality and multiple cavernous hemangiomas.

The patient underwent antibiotic therapy for 7 days, with clinical improvement but no change of the thoracic image. At this point, numerous differential diagnoses were taken into account (respiratory tract abnormality, tumor mass) and a CT-scan of the thorax was performed. It demonstrated Morgagni's hernia with liver thoracic dislocation (fig. 3).

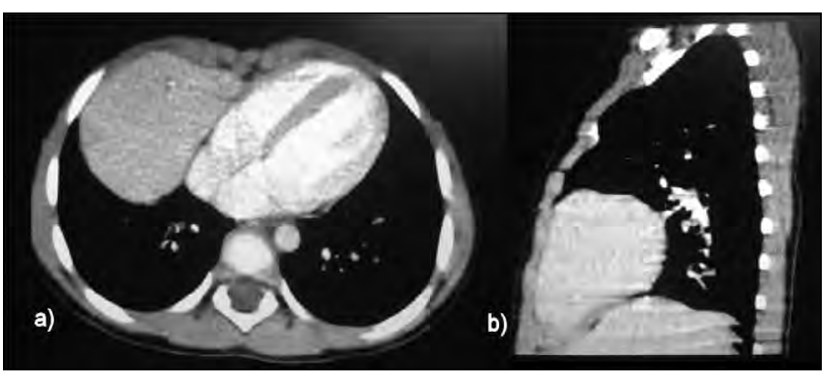

FIGURE 3. CT scan of the thorax (a) transverse section, (b) sagittal section

The patient underwent a single contract barium enema that proved no bowel loop herniated in the thorax (fig. 4).

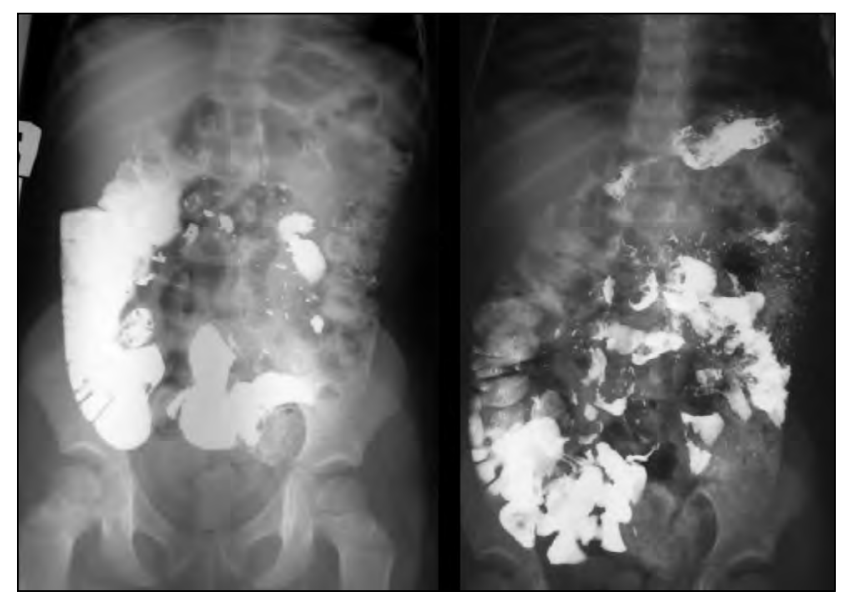

FIGURE 4. Irigography: No bowel loop herniated in the thorax
At this point in time, the surgical examination ruled out the risk of bowel obstruction and did not consider an immediate surgical therapy required. The surgical option was revisited three years later and set the indication for corrective surgery, as signs of pulmonary hypertension started to develop.

\section{DISCUSSIONS}

The most common clinical feature of $\mathrm{CDH}$ is respiratory distress (cyanosis, retractions, grunting respiration) in the first 24 hours due to lung hypoplasia, which occurs mainly on the same side as the hernia. Nevertheless, there are some types of diaphragm defects, such as Morgagni hernia, without any clinical manifestations in the newborn period. In our case, the patient required intensive care in the neonatal period, being prematurely born. The opacity seen on thoracic x-ray was at that point considered right pulmonary hemorrhage; comparing the two images (fig. 1 and 2a), the similarity is obvious. Other clinical findings of CGH include a scaphoid abdomen, barrel-shaped chest, intestinal sounds in the thorax and fast heart rate. Craniofacial dysmorphism, extremity abnormalities or spinal dysraphism can be found in syndromic $\mathrm{CDH}$ $(1,7,8)$. None of these were described in our case.

$\mathrm{Up}$ to one-third of CDMH cases are reported clinically asymptomatic despite the congenital nature of the illness. The condition may present with recurrent chest infections, gastrointestinal symptoms or nonspecific signs. Gastrointestinal presentation in rare and usually obstructive in nature due to the incarceration of bowel loop (especially the colon) in the hernia sac $(3,8)$. In the case presented the diagnosis was incidentally made as the patient developed persistent respiratory signs due to acute, unrelated, respiratory pathology and a chest x-ray was done.

Among the complications associated with $\mathrm{CDH}$ are pulmonary hypertension, left ventricular dysfunction, chronic lung disease, gastroesophageal reflux or underdeveloped intestines. Because most children with serious lung problems are most likely to have growth problems, failure to thrive is usually present, accompanied by developmental delays (7). Our patient exhibited no complications by the age of 6 . When signs of pulmonary hypertension started to develop, the surgical recommendation shifted to a more aggressive approach.

A redoubtable complication, which appears in right-sided hernias, is hepatic pulmonary fusion, an extremely rare condition (only 14 cases in litera- 
ture, most of them in the neonatal period/ infancy). Hepatic and pulmonary tissue fuse by a fibrous band, broncho-biliary fistula or anomalous drainage from the right lung to the intrahepatic inferior vena cava are findings related to HPF (9).

Prenatal investigations in the second trimester detect approximatively $60 \%$ of affected fetuses with right-sided hernia and $80 \%$ of those with leftsided CDH. Obstetric ultrasound performed during routine screening or during investigations for polyhydramnios (which can reportedly complicate up to $80 \%$ of pregnancies with $\mathrm{CDH}$ fetuses) is the primary diagnostic method. MRI is useful for detecting atypical thoracic lesions or assessing lung volumes (estimated fetal lung volume and the amount of herniated viscera are critically prognostic indicators). Color flow Doppler identifies right-sided hernias, which can be difficult to detect on ultrasound examination due to the similar echogenicity of lung and liver. It can also suggest liver herniation by demonstrating abnormal position of the umbilical and portal vein $(1,2,10)$. The CDMH remained undiagnosed prenatally in our case, although the pregnancy was continuously monitored with repeated US investigations. Postnatal diagnosis relies on clinical presentation - diminished breath sounds ipsilateral to the side of the hernia, displacement of heart sound contralateral to the hernia - as well as chest X-ray, which shows mediastinal shift and visible bowl gas above the diaphragm (1). No bowel was herniated in this case, so the diagnosis was overlooked repeatedly in the neonatal period.

Late diagnosis is characterized by the presence of chest mass on the radiologic exam, as in our case, gastric volvulus or bowel obstruction. Other important findings include left ventricle hypoplasia in left-sided hernias and pleural effusions in rightsided ones (6).

Surgical therapy is performed in an open or minimally invasive manner. In most cases abdominal approach is preferred instead of thoracoscopic methods, more likely if there are any anomalies associated with $\mathrm{CDH}$ (ex: intestinal malrotation). For $\mathrm{CDMH}$, surgical repair is recommended on a semiurgent basis to avoid morbidities $(2,8)$. The second surgical opinion is in our view according to most of literature data. Although the patient does not have risk of bowel obstruction, other comorbidities related to thoracic dislocation of the liver must be decisive in the therapeutic decision.

Recent data reported a mean survival rate of $63 \%$ for $\mathrm{CDH}$ children. Isolated $\mathrm{CDH}$ has generally a better prognostic than $\mathrm{CDH}$ complicated by numerous anomalies. There are three major predictors of outcome: associated anomalies (especially heart disease), the degree of pulmonary hypoplasia and liver herniation. Moreover, right-sided hernias have a greater mortality rate than left-sided ones $(1,2,10)$.

\section{CONCLUSIONS}

Diaphragmatic hernia is a challenging diagnosis to be made. Although a congenital condition, it may be asymptomatic for a long time and a high degree of suspicion is required, especially in the right variant for which most of the data is based on case reports and case series.

Conflict of interest: none declared Financial support: none declared

\section{REFERENCES}

1. Longoni M, Pober BR, High FA. Congenital Diaphragmatic Hernia Overview. 2006 Feb 1 (Updated 2019 Mar 28). In: Adam MP, Ardinger HH, Pagon RA, et al., editors. GeneReviews $® 2017$ (Internet). Seattle (WA): University of Washington, Seattle; 1993-2019.Available from: https://www.ncbi.nlm.nih.gov/books/NBK1359/;

2. Chandrasekharan PK, Rawat M, Madappa R et al. Congenital Diaphragmatic hernia - a review. Matern Health Neonatol Perinatol. 2017;3:6;

3. Slepov O, Kurinnyi S, Ponomarenko O et al. Congenital retrosternal hernias of Morgagni: Manifestation and treatment in children. Afr J Paediatr Surg. 2016;13:57-62;

4. Sarkar A, Hajra GSD. Right Sided Congenital Diaphragmatic Hernia A Case Report With A Brief Review. J. Anat. Soc. India. 2012;61(1):53-56;

5. Almaawi A, Prasad DRK, Waqasi Z et al. Right Sided Congenital Diaphragmatic Hernia, an Operative Challenge. J Pediatr Neonatal Care. 2015;2(3):00074;
6. Sardescu G, Sbârcea A, Cîrstoveanu C. Right-sided congenital diaphragmatic hernia - case report. MEDICHUB MEDIA. Perinatologia 2017, 1(3) DOI: 10.26416/Peri.1.3.2017;

7. Boston Children's Hospital. Congenital Diaphragmatic Hernia. (Internet). http://www.childrenshospital.org/conditions-and-treatments/ conditions/c/congenital-diaphragmatic-hernia;

8. Al Shanafey S. Morgagni Diaphragmatic Hernia in Children: One Center's Experience. World Journal of Surgery and Surgical Research. 2018;1:1021;

9. Laamiri R, Belhassen S, Ksia Amina et al. Right Congenital Diaphragmatic Hernia Associated With Hepatic Pulmonary Fusion: A Case Report. J Neonatal Surg. 2016; 5(3):35;

10. Skarsgard ED, Harrison MR. Congenital Diaphragmatic Hernia: The Surgeon's Perspective. Pediatrics in Review. 1999;20(10):71-78. 